\title{
Intracellular Symbiotic Bacteria of Camponotus textor, Forel (Hymenoptera, Formicidae)
}

\author{
Manuela O. Ramalho' ${ }^{1}$ (D) Cintia Martins $^{2} \cdot$ Larissa M. R. Silva $^{1}$. \\ Vanderlei G. Martins ${ }^{1} \cdot$ Odair C. Bueno $^{1}$
}

Received: 17 November 2016 / Accepted: 19 January 2017 / Published online: 6 March 2017

(C) Springer Science+Business Media New York 2017

\begin{abstract}
This study focuses on the weaver ant, Camponotus textor, Forel which occurs in some areas of the Brazilian Cerrado and Atlantic Forest, and its symbionts: Blochmannia, an obligate symbiont of Camponotus, and Wolbachia, known for causing reproductive alterations in their hosts. The main goal of this study was to investigate the presence, frequency of occurrence, and diversity of Wolbachia and Blochmannia strains in C. textor colonies. We found high infection rates $(100 \%)$ and the occurrence of at least two distinct strains of Blochmannia (H_1 or H_7) in the same species. The observed haplotype variation within a single species may result from the high mutation rate of the symbiont. Similarly, the Wolbachia was found in all colonies with different rates of infections and a new strain (supergroup A) was deposited in the MLST database. The diversity found in the present study shows that there is still much to explore to understand about these symbiotic interactions.
\end{abstract}

Manuela O. Ramalho

manuramalho2010@gmail.com

Cintia Martins

martins.c@ufpi.edu.br

Larissa M. R. Silva

larissamedski@yahoo.com.br

Vanderlei G. Martins

martv@uol.com.br

Odair C. Bueno

odaircb@rc.unesp.br

1 Universidade Estadual Paulista "Júlio de Mesquita Filho" UNESP - Campus Rio Claro, Biologia, CEIS, Av. 24A, 1515, Bela Vista, Rio Claro, SP 13506-900, Brazil

2 Universidade Federal do Piauí - Campus Ministro Reis Velloso, Av. São Sebastião, 2819, 64.202-020, Parnaíba, Piauí, Brazil
Keywords Endosymbiont - Camponotini - Weaver ant . Wolbachia $\cdot$ Blochmannia

\section{Introduction}

Symbiotic relationships between insects and bacteria occur in virtually all orders, including Hymenoptera, and may be divided into primary (obligate) and secondary (facultative) interactions, both types have been identified in Formicidae $[1,2]$. Obligate endosymbionts are the result of an ancient association with the host; they usually live inside specialized cells called bacteriocytes and contribute to ant nutrition. As a result of this association, the bacterial genome, which is vertically transmitted, may shrink [3]. Blochmannia is an example of an obligate symbiont that is commonly found in Camponotus species in the Northern Hemisphere [4].

In contrast, facultative symbionts are characterized by a more recent association and may be transmitted vertically or horizontally [5]. Wolbachia, for example, stands out for interfering in the reproduction of its hosts, but its role in the worker caste of ants is unknown. According to recent estimates, between 20 and 35\% of arthropods are infected with Wolbachia [6]. Wolbachia has a large diversity of strains, which are divided into supergroups (A-F); the strains found in insects belong exclusively to supergroups A and B. Traditionally, Wolbachia infections were detected with the wsp gene [7]; however, due to its high rate of recombination [8] and strong selection for diversification [9] a multilocus sequence typing (MLST) approach has gained popularity [10].

In studies of intracellular bacteria, the term cospeciation is often used, and these associations between insects (host) and bacteria are well documented in the literature. 
Examples include aphids, the tsetse fly, cockroaches, and their respective symbionts [11-13]. In ants, this type of interaction also results in a high degree of congruence between host and symbiont phylogenetic trees, indicating the occurrence of parallel diversification and of maternal transmission of the infection. However, the geographic distribution may only partially reflect the congruence between host and symbiont phylogenetic trees [14].

One of the best known genera of ants for having symbiotic relationships with bacteria is Camponotus Mayr, 1861 $[15,16]$ and in a recent study by Bronw and Wernergreen [17] found that 95-98\% of bacteria found in Camponotus chromaiodes were Blochmannia and Wolbachia, but most studies of the microbiota and host are restricted to the Northern Hemisphere.

Camponotus textor, Forel as well as 14 other species of ants distributed around the world, is known as a weaver ant because it uses silk to construct its nests [18]. Although the existing literature suggests that this species is common in the forests of Central and South America, its precise distribution is not fully known [19]. In general, published works describe only behavioral traits, mostly related to nest construction using the silk produced by their larvae [20].

The taxonomy of the species is complicated. C. textor is often mistaken for $C$. senex (Smith) due to their morphological similarities. Recently, however, Ramalho et al. [21] distinguished the two species based on molecular data and ecological traits of specimens of both species collected in the Neotropics. Their results corroborate with Longino [22], who considers them as two separate species. Although $C$. textor has elaborate behaviors, little is known about its feeding habits, biology, ecology, and interactions with other organisms [20, 23, 24].

Other few studies involving ants and endosymbionts from the Neotropical region have already shown how diverse these associations are [25, 26]. Since the descriptions of primary and secondary symbionts in Camponotus are based on species from the Northern Hemisphere, the goal of this study was to investigate the presence, frequency of occurrence, and strain diversity of Wolbachia and Blochmannia in C. textor colonies, which are ants exclusively Neotropical, and evaluate the diversity of these endosymbionts.

\section{Materials and Methods}

\section{Collection, Identification, and Total DNA Extraction}

Camponotus textor workers were collected from eight localities in different regions of Brazil with either typical Cerrado or Atlantic Forest vegetation: Rio Claro, SP

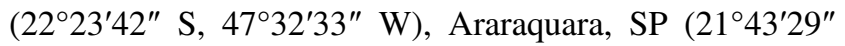

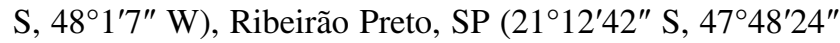

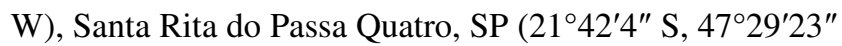
W), São João da Boa Vista, SP (21 ${ }^{\circ} 58^{\prime} 10^{\prime \prime}$ S, 46 47'56" W), Uberlândia, MG (two colonies: $18^{\circ} 53^{\prime} 10^{\prime \prime} \mathrm{S}, 48^{\circ} 15^{\prime} 39^{\prime \prime}$ $\mathrm{W}$, and 18 $\left.53^{\prime} 1^{\prime \prime} \mathrm{S}, 48^{\circ} 15^{\prime} 34^{\prime \prime} \mathrm{W}\right)$, and Ilhéus, BA (14 $\left.18^{\prime} 45^{\prime \prime} \mathrm{S}, 39^{\circ} 53^{\prime} 13^{\prime \prime} \mathrm{W}\right)$. The collected material was preserved in $80 \%$ ethanol and kept at $-20^{\circ} \mathrm{C}$ until DNA extraction. Specimens were identified by Dr. Jacques Delabie and deposited in the collection of CEPLAC, Ilhéus, BA (accession number 5692).

Total DNA was extracted from eight individual workers of each colony and preserved in $80 \%$ ethanol [19, 27]. We used primers Bloch16S-462F and Bloch16S-1299R [16] to screen for Blochmannia. We used primers wsp81f and wsp691r for the initial detection of Wolbachia [7, 28] and EF1 $\alpha-532 f$ and EF1 $\alpha-610$ r [29] as positive controls. The amplification was performed using Taq DNA Polymerase, Recombinant (Invitrogen), following the protocol of the manufacturer. We used the thermal cycler parameters recommended by Baldo et al. [30] and Wernegreen et al. [16] to identify Wolbachia and Blochmannia, respectively.

Purification of the PCR product was performed using the GFX PCR DNA and Gel Band Purification kit (GE Healthcare). Samples were quantified in the Thermo Scientific NanoDrop 2000 (Uniscience) and sequenced using the BigDye Terminator v3.1 reagent kit (Applied Biosystems). Sequence reading was carried out in a 3130 Genetic Analyzer automated sequencer (Applied Biosystems).

\section{Analyses: Blochmannia}

After the sequences were edited, multiple Blochmannia infections were detected. As a result, cloning with the pGEM-T Vector System I (Promega) was required to isolate each sequence; we followed the protocol provided by the manufacturer. Miniprep followed Zhou et al. [31], and the sequencing reactions were prepared as described before. Samples that have succeeded in sequencing were deposited in GenBank (accession codes KX212263-KX212309, Ilhéus, Rio Claro, São João da Boa Vista and Santa Rita do Passa Quatro colonies). A haplotype network was constructed using sequences with highest similarity found in Genbank (E-values of 0.0 and $98 \%$ similarity with "Candidatus Blochmannia ulcerosus" AY334375.1, "Candidatus Blochmannia laevigatus" AY334370.1, and "Candidatus Blochmannia herculeanus" AJ250715.1) with the medianjoining method in Network 4.5.1.0 [32].

To test whether there is geographic correlation of the colony with the several strains of Blochmannia, we use the Mantel test available in "vegan" package [33] of R software [34]. The geographical coordinates of the colonies were transformed to metric UTM using the "rgdal" package [35], and the genetic distance of each Blochmannia sequence 
was calculated using the Kimura 2-parameter model [36] in PAUP 4.0 [37].

\section{Analyses: Wolbachia MLST}

The sequences generated were edited and aligned using BioEdit sequence alignment editor [38] and ClustalW [39]. The sequences obtained with the $w s p$ primers allowed us to determine if the Wolbachia infections were single or multiple. If a single infection was confirmed, the Wolbachia MLST approach was initiated following the single infection protocol available at the MLST website (http://pubmlst. org/wolbachia/). All the analyses were run in triplicate (three different workers per colony). Double-infected colonies were excluded from the analyses. The alleles of each gene were compared one at a time with those deposited on the MLST database, and the sequence types (ST) were later confirmed through the concatenated analysis. The sequences from the $w s p$ primers were compared with others in the same database [30].

The dataset was partitioned into the different genes, and an appropriate model of sequence evolution was chosen using the Akaike Information Criterion in ModelTest v3.06 [40]. The models selected were GTR_I_G for gat $B$ and $w s p$, and GTR_G for coxA, $h c p A$, ftsZ, and $f b p A$. Phylogenetic reconstruction based on Bayesian analysis was carried out in MrBayes 3.1.2 [41] by running a 1,000,000-generation Markov chain and sampling every 1000 generations. The first $25 \%$ of the trees were discarded as burnin, and the probability values were calculated using the remaining trees. All the Formicidae STs in the MLST database were used for the comparative analysis. Since there is only one Formicidae B strain, other insects were added to compare and confirm the supergroups. The D strain was used as the outgroup.

\section{Results}

\section{Blochmannia}

All Camponotus specimens analyzed were positive for Blochmannia, with a total of 47 clones distributed in 22 different haplotypes, with 508-bp of the 16S rRNA region (Fig. 1). The network analysis revealed that Blochmannia haplotypes found in $C$. textor are distant (46 different nucleotides) from other Blochmannia sequences in Camponotus previously deposited in the database. As these Blochmannia from other Camponotus were selected to possess the highest similarity with the present study, it emphasizes how different the Blochmannia found in C. textor is.

In addition, there was more than one Blochmannia strain per worker, and these strains were not exclusive to a given geographic location. For example, haplotype 1 (H_1) was found in all locations and haplotype 7 (H_7) was only absent from São João da Boa Vista. There was no correlation between the Blochmannia genetic distances and the geographic locations of the colonies using the Mantel test $(r=0.023, P=0.3)$. In addition, haplotype 1 (H_1) was the most frequent, followed by haplotype 7 (H_7), the two differed by a single nucleotide. The $\mathrm{H} \_1$ haplotype differs by only one nucleotide of the following haplotypes: H_3, H_22, H_5, H_4, H_21, H_14, H_13, by two nucleotides: H_2, H_18, H_17, H_15, H_19, H_20; by three nucleotides: H_12, H_16; and finally $\mathrm{H} \_6$ differ by five nucleotides. The H_7 haplotype differs by only one nucleotide of the following haplotypes: $\mathrm{H} \_8, \mathrm{H} \_11$ and $\mathrm{H} \_9$, and by two nucleotides $\mathrm{H}_{-}$10. All these mutations happened in different loci.

The haplotype network suggests that there are two distinct Blochmannia lineages (H_1 and H_7) in the ant population, and that the other haplotypes derive from $\mathrm{H} \_1$ and H_7 (Fig. 1). This could explain the co-occurrence of $\mathrm{H}_{-} 7$ with $\mathrm{H} \_8, \mathrm{H} \_9$, and $\mathrm{H} \_10$, and the co-occurrence of $\mathrm{H} \_1$ with $\mathrm{H} \_2$ and $\mathrm{H} \_3$. Except the haplotype $\mathrm{H} \_22$ being cooccurring with $\mathrm{H}_{-} 7$, all other haplotypes follow the pattern of the co-occurrence happens between haplotypes deriving from. For many others haplotypes found, we could not exclude the possibility of sequencing artifacts. Therefore, we decided to focus our discussions in these haplotypes that were repeatedly detected in different ant specimens (H_1 and H_7).

\section{Wolbachia}

Wolbachia was detected in all colonies analyzed with the wsp gene, but its rates of infection varied: $75 \%$ in Rio Claro, 37.5\% in Santa Rita do Passa Quatro, 87.5 and 80\% in Uberlândia I and II, and 100\% in Araraquara, Ribeirão Preto, Ilhéus, and São João da Boa Vista. The electropherogram revealed both single and double infections. The only colonies with single infections were in Santa Rita do Passa Quatro (SP) and São João da Boa Vista (SP).

The triplicate MLST sequences from the single-infected colonies were compared with the sequences deposited in the Wolbachia MLST database. There was no variation within the species; in other words, all alleles for all individuals were identical. However, we found a new allele, $\operatorname{cox} A$ allele 185, and a novel ST, ST347, was deposited in the MLST database as a consequence. The sequences generated by $w s p$ were included in an additional analysis, which revealed hypervariable regions (HVR1: allele 37, HVR2: allele 38, HVR3: allele 41, and HVR4: allele 37) and 100\% similarity with wsp allele 58 .

The Bayesian inference analysis (using Bayesian posterior probabilities, $\mathrm{BPP}$ ) of the 42 concatenated sequences 
Fig. 1 Blochmannia haplotypes in Camponotus textor. a Haplotype network showing the higher frequency of $\mathrm{H}_{-}$1, followed by H_7. b Distribution of the different haplotypes from each analyzed individual. The haplotype size represents the frequency found, and the point in red was added by the software as hypothetical haplotype. ${ }^{*} C$. textor from this study. (Color figure online)

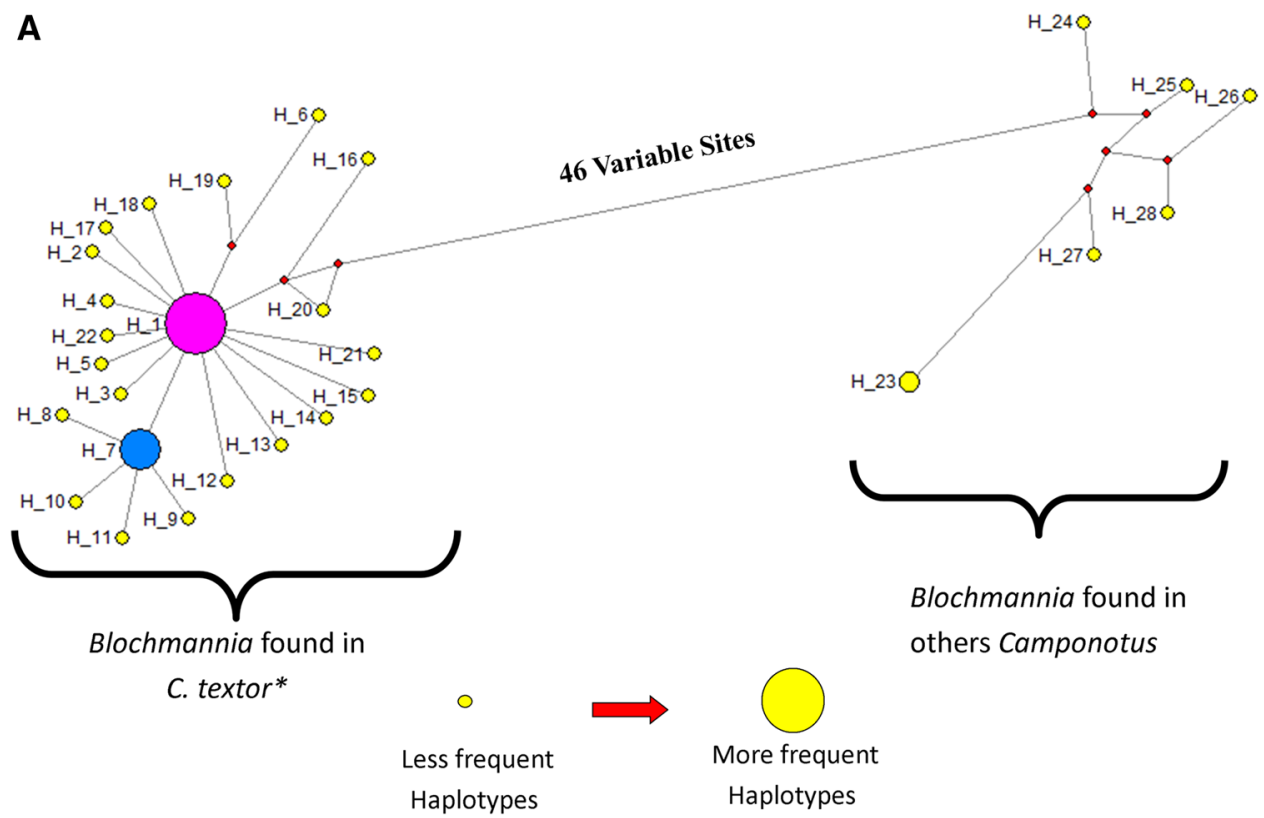

B

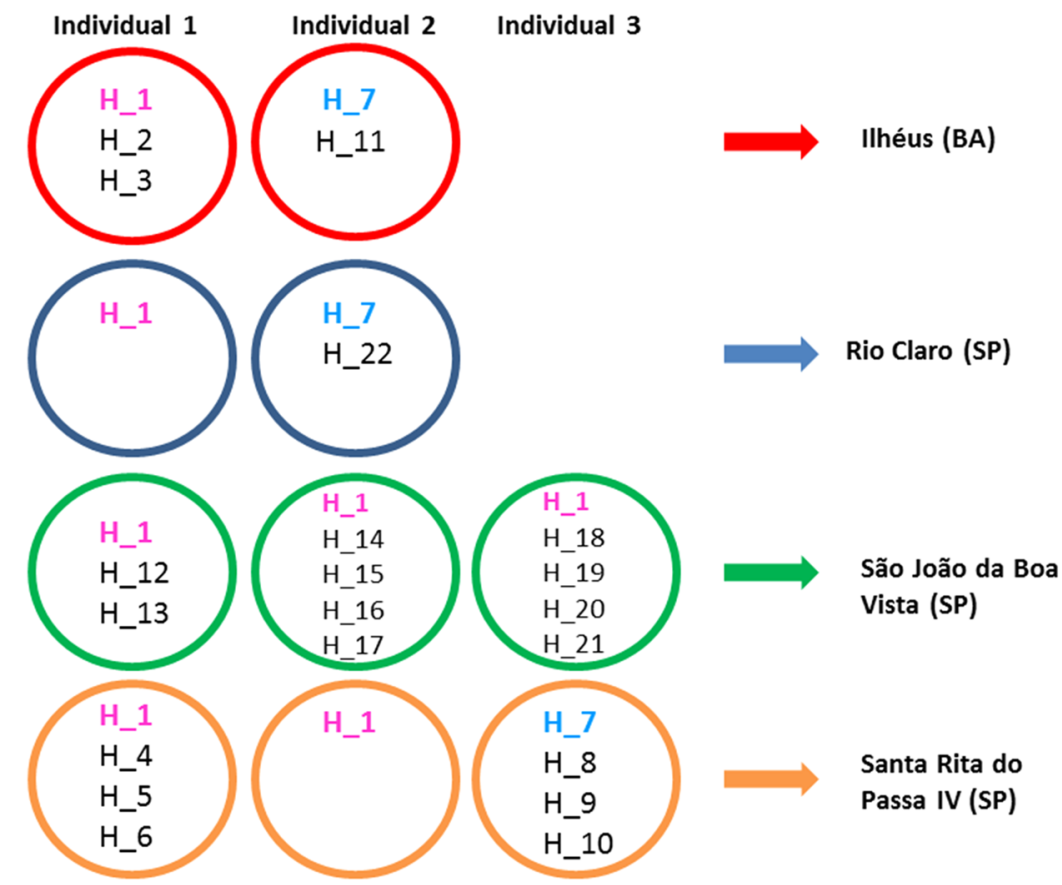

in different supergroups revealed that ST347 was more closely related to supergroup A STs, thus characterizing this new strain (Fig. 2). However, the strain found in the present study is separated from the strains from North America and the Old World. Combined with ST45, this new strain forms a strongly supported clade $(B P P=90)$ that is separated from other supergroup A strains. Supergroup A and supergroup B were separated by 100 bp.

\section{Discussion}

\section{Blochmannia}

Corroborating the findings of Sameshima et al. [15] and Wernegreen et al. [16], all analyzed individuals had Blochmannia suggesting that the bacteria are fixed within populations of species in the ant genus Camponotus, as 


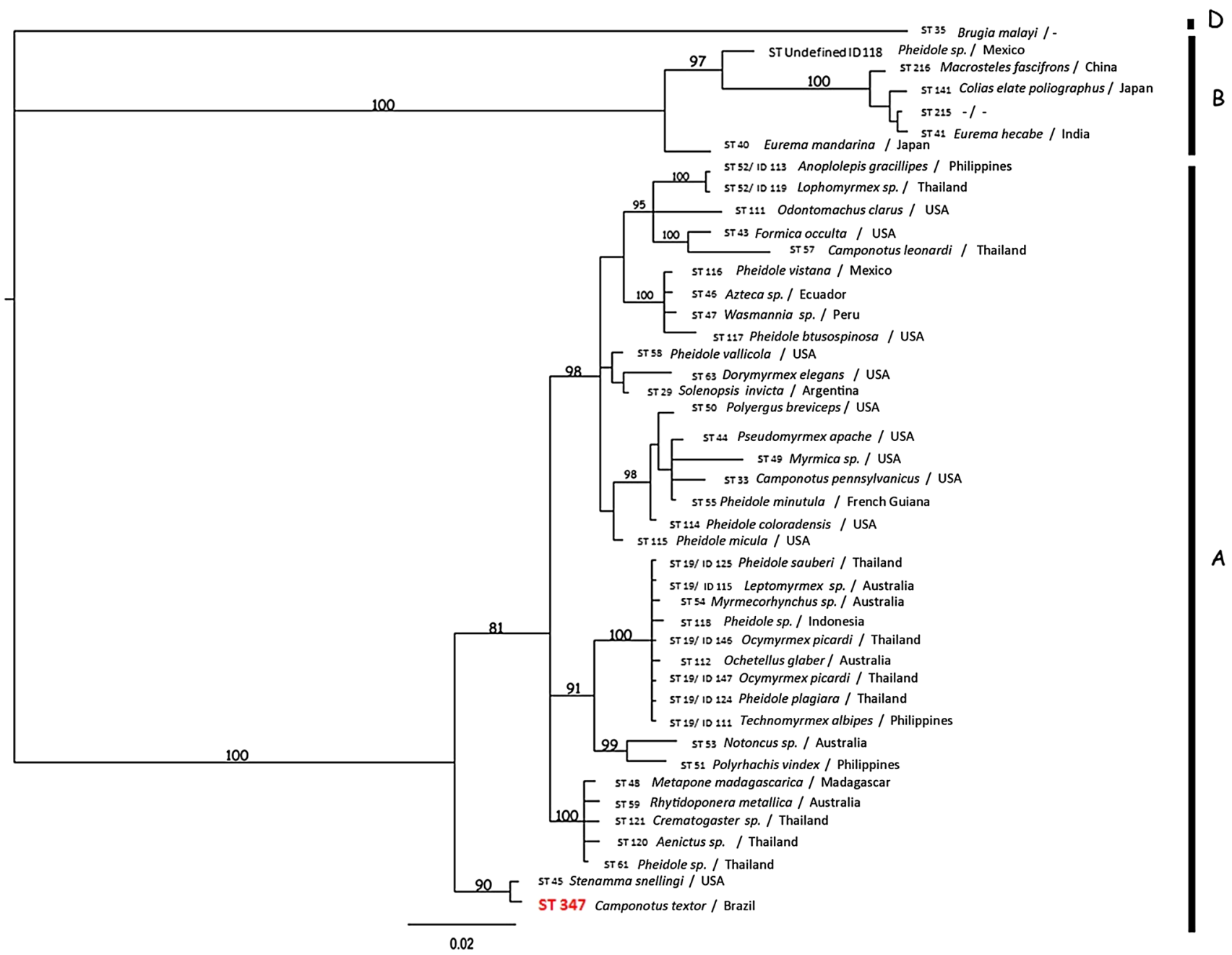

Fig. 2 Bayesian inference analysis of the sequences in the Wolbachia with the host/location available in MLST database. The Wolbachia strain found in Camponotus textor and identified through this work

observed in Formica truncorum and its obligate endosymbiont Wolbachia [42]. Additionally, Blochmannia strains found in the present work were very different to "Candidatus Blochmannia ulcerosus" (AY334375.1, USA), "Candidatus Blochmannia laevigatus" (AY334370.1, USA), and "Candidatus Blochmannia herculeanus" (AJ250715.1, USA), which confirms the high interspecific diversity. We believe that this huge difference could be because (I) there are few studies in South America that analyzed the diversity of this endosymbiont, and (II) this bacterium has a high rate of mutation [43]. Besides that, there was no correlation between the geographical location and strain similarity based on the intraspecific haplotype network of the Blochmannia sequences found in C. textor, i.e., shared strains occurred in different geographical regions (Fig. 3).

The intracellular endosymbionts that live in bacteriocytes are vertically transmitted [44]. The fact that the is highlighted (ST347), and belongs to the supergroup A clade. The symbol "-" means that the information was unavailable

bacteria are located inside a specialized organ associated with female reproductive tissues suggests that the speciation processes of the host and its endosymbiont are interconnected $[45,46]$. Phylogenetic congruence suggests the absence of horizontal transfer [43], contrasted to the recurrent recombination among strains of free-living bacteria [47]. The present study analyzed different colonies from different locations and showed the same pattern of diversity of Blochmannia (H_1 and H_7, in different locations). Therefore, there is no evidence of horizontal transmission of Blochmannia among these populations of C. textor, suggesting that the long-distance migration of the ants happened in the past and that the common ancestor of this ants has been carrying these strains ever since $[15,16,44]$.

The high intraspecific diversity of Blochmannia haplotypes in this species should also be noted. Based on the frequencies observed, we cannot exclude the possibility of 
Fig. 3 Distribution map of Blochmannia haplotypes found in Camponotus textor. Haplotype 1 (H_1) was the most common and it is highlighted in pink. Haplotype 7 (H_7) is highlighted in blue. Note that there are colonies with the presence of both haplotypes. Locations where we confirmed the presence of Blochmannia, but we cannot define the haplotype by the technique of cloning, are highlighted in gray. (Color figure online)

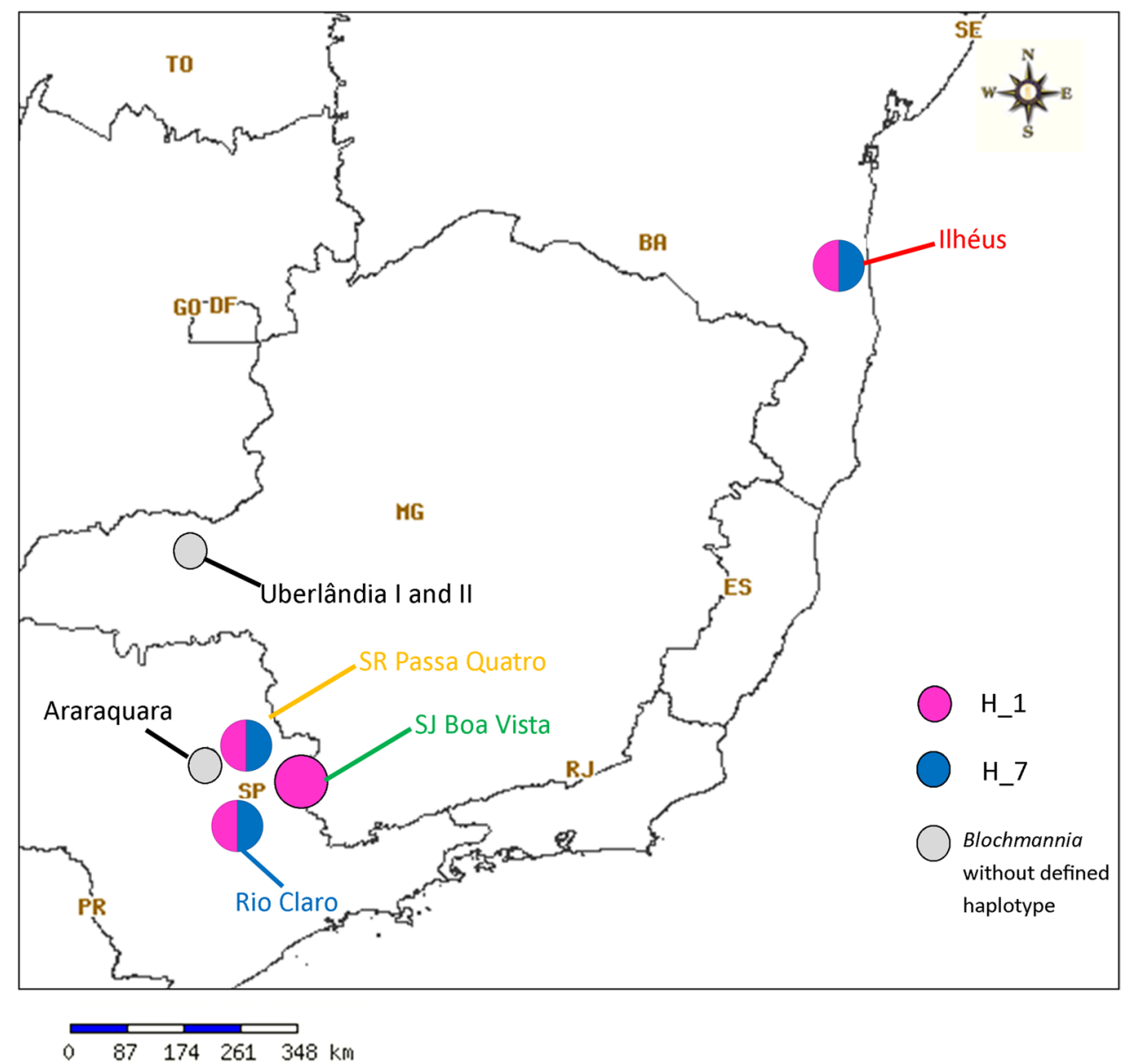

at least two distinct haplotypes coexisting in the same host species. Degnan et al. [43] observed a high mutation rate in Blochmannia, which might explain the occurrence of haplotype variation within a single species. Nevertheless, we should consider three hypotheses: (1) this diversity is a sequencing artifact; (2) there are multiple copies of $16 \mathrm{~S}$ in the Blochmannia genome; and (3) there are multiple strains of Blochmannia in $C$. textor. Given the high frequency of haplotypes identified in this study, the possibility of a sequencing artifact may be rejected, at least for H_1 and H_7, which were frequently found in different colonies. The second hypothesis merits further investigation. Because we did not analyze the whole genome of Blochmannia, we do not know if there are multiple copies of the $16 \mathrm{~S}$ genome. However, we checked whether there is precedence in the publicly available genome sequence of the Blochmannia endosymbiont from C. obliquus strain 757 , GenBank accession \# NZ_CP010049, and it was found in only one copy of the $16 \mathrm{~S}$ gene [48]. Therefore, we also believe that this hypothesis does not apply to $C$. textor. The third hypothesis is the most plausible; although we sampled different colonies from different locations, we observed the same two haplotypes in each one of them (either H_1 or
H_7). Due to the high mutation rate of Blochmannia, it is possible that these diversified strains of Blochmannia had already been present in the ancestor of $C$. textor, and that they spread along with their host species as the geographic range of $C$. textor expanded.

In our study, a pattern emerged where every worker analyzed harbored either haplotype H_1 or H_7 (Fig. 1b). Interestingly, however, these two dominant haplotypes never co-occurred, that is, with our sampling protocol we were unable to find haplotypes $\mathrm{H}_{-} 1$ and $\mathrm{H} \_7$ in the same individual. More studies are needed to address the possibility of incompatibility or competition between these haplotypes. It is important to note that little is known of the general biology of this host ant, and in particular if the colonies are polygynous or monogynous. If this species is monogynous, the single queen may harbor both haplotypes, excluding the possibility of incompatibility or competition. However, an additional question may be raised: why would these multiple strains not be quickly lost due to genetic drift? Genetic drift does not prevent the co-occurrence of multiple strains of Wolbachia within the same individual [25, 26, 49, 50], and we believe the same could be the case for Blochmannia, explaining its diversity. If functional 
divergence has occurred, akin to the recent diversification of symbionts in cicadas reported by Campbell et al. [51], more studies will be needed to understand the diversity of Blochmannia in Camponotus sp.

\section{Wolbachia}

Camponotus textor had a high rate of Wolbachia infection compared to Solenopsis spp. from the same region [25], suggesting that the bacteria may be at or near fixation, as suggested by Wenseleers et al. [42] in F. truncorum, Fabricius. However, the ST and the wsp gene did not vary among different colonies, suggesting that the Wolbachia infection may have occurred a long time ago in the common ancestor of the populations. According to Watanabe et al. [52], there are three possible explanations for the presence of similar strains of Wolbachia in related species: vertical transmission by a common ancestor [53], horizontal transmission [54], and introgressive hybridization between the hosts $[55,56]$.

Introgressive hybridization may be discarded because we are reporting similar strains of Wolbachia within a species $C$. textor. Therefore, the observed distribution of Wolbachia may be caused by(a) vertical transmission by a common ancestor, maintained despite the geographic separation of the colonies, or (b) horizontal transmission induced by similar host-parasitoid or predator-prey interactions [52]. These two hypotheses are also supported by the results of Salunke et al. [57], who used MLST to study Wolbachia in butterflies.

Phylogenetically, several strains of Wolbachia have been detected in different species of ants using the MLST methodology, and the great majority belongs to supergroup A and the ST347 strain. The distribution of the supergroups $(\mathrm{A}, \mathrm{B}$, and $\mathrm{D})$ in the haplotype network and the reconstructed phylogeny confirm that the supergroups are completely separated. Haplotype 31 and haplotype 10 (which are equivalent to ST347 and ST45, respectively) were closely related, both in the network and in the phylogenetic tree, but they are distant from the other strains in supergroup A. The similarity among the variants in family Formicidae was also confirmed through MLST, indicating that there are differences between the strains found in ants and those from other insects, and between the variants in the New World and the Old World [6].

\section{Conclusion}

Research into symbiotic interactions of ants of the genus Camponotus often focuses on Blochmannia, but the actual diversity of the bacterial community of this genus is still unknown. In the general context, we were able to find at least two strains of Blochmannia present in the same species of $C$. textor, an ant occurring only in the Neotropical region. One possible explanation for the occurrence of these strains could be the high mutation rate of Blochmannia. In the same species, the high infection rate was also observed for Wolbachia, and a new strain was deposited in the MLST database. However, these new ST and wsp genes were the same for all $C$. textor colonies analyzed, suggesting that the Wolbachia infection occurred in the past in the common ancestor of these populations, before the colonies split. New studies with $C$. textor using next-generation sequencing technologies are needed to obtain more data on the role of symbiotic relationships and their implication for the biology of the host.

Acknowledgements We thank all our colleagues for their assistance: Dr Kleber Del-Claro, Dr Jacques Hubert Charles Delabie, Dr Viviane Cristina Tofolo Chaud, Dr Maria Santina Castro Morini, Dr Ana Carolina Marchiori, Dr Cynara de Melo Rodovalho, and Dr Corrie $\mathrm{S}$. Moreau. We thank reviewers and editors who provided valuable suggestions for elaboration in this study. We thank Marilia de Oliveira Ramalho for editing images. This work supported by the CAPES Foundation, Brasilia, Brazil [Grant Number 007343/2014-00].

Funding This work supported by the CAPES Foundation, Brasilia, Brazil [Grant Number 007343/2014-00].

\section{Compliance with Ethical Standards}

Conflict of interest The authors declare that they have no conflict of interest.

\section{References}

1. Dasch GA, Weiss E, Chang K (1984) Endosymbiosis of insects. Bergey's Man Syst Bacteriol 1:811-833

2. Zientz E, Feldhaar H, Stoll S, Gross R (2005) Insights into the microbial world associated with ants. Arch Microbiol 184:199206. doi:10.1007/s00203-005-0041-0

3. Williams LE, Wernegreen JJ (2010) Unprecedented loss of ammonia assimilation capability in a ureaseencoding bacterial mutualist. BMC Genomics 11:687. doi:10.1186/1471-2164-11-687

4. Blochman F (1882) Über das vorkommen von bakterienähnlichengebilden in den geweben und eiernverschiedenerinsekten. Zentbl Bakteriol 11:234-240

5. Oliver KM, Degnan PH, Burke GR, Moran NA (2010) Facultative symbionts in aphids and the horizontal transfer of ecologically important traits. Annu Rev Entomol 55:247-266. doi:10.1146/annurev-ento-112408-085305

6. Russell JA, Funaro CF, Giraldo YM, Goldman-Huertas B, Suh D, Kronauer DJC, Moreau CS, Pierce NE (2012) A veritable menagerie of heritable bacteria from ants, butterflies, and beyond: broad molecular surveys and a systematic review. PLoS ONE 7:e51027. doi:10.1371/journal.pone.0051027

7. Zhou W, Rousset F, O'Neill S (1998) Phylogeny and PCR-based classification of Wolbachia strains using wsp gene sequences. Proc R Soc Lond B 265:509-515 
8. Baldo L, Lo N, Werren JH (2005) Mosaic nature of the Wolbachia surface protein. J Bacteriol 187:5406-5418. doi:10.1128/ JB.187.15.5406-5418.2005

9. Baldo L, Bartos JD, Werren JH, Bazzocchi C, Casiraghi M, Panelli S (2002) Different rates of nucleotide substitutions in Wolbachia endosymbionts of arthropods and nematodes: arms race or host shifts? Parassitologia 44:179-187

10. Paraskevopoulos C, Bordenstein S, Wernegreen JJ, Werren JH, Bourtzis K (2006) Toward a Wolbachia multilocus sequence typing system: discrimination of Wolbachia strains present in Drosophila species. Curr Microbiol 53:388-395. doi:10.1007/ s00284-006-0054-1

11. Aksoy S (1995) Molecular analysis of the endosymbionts of tsetse flies: 16S rDNA locus and over-expression of a chaperonin. Insect Mol Biol 4:23-29. doi:10.1111/j.1365-2583.1995. tb00004.x

12. Bandi C, Sironi M, Damiani G, Magrassi L, Nalepa CA, Laudani U, Sacchi L 1995 The establishment of intracellular symbiosis in an ancestor of cockroaches and termites. Proc R Soc Lond B 259:293-299

13. Baumann P, Baumann L, Lai CY, Rouhbakhsh D, Moran NA, Clark MA (1995) Genetics, physiology and evolutionary relationships of the genus Buchnera: intracellular symbionts of aphids. Annu Rev Microbiol 49:55-94

14. Sauer C, Stackebrandt E, Gadau J, Hölldobler B, Gross R (2000) Systematic relationships and cospeciation of bacterial endosymbionts and their carpenter ant host species: proposal of the new taxon Candidatus Blochmannia gen. nov. Int J Syst Evol Microbiol 50:1877-1886. doi:10.1099/00207713-50-5-1877

15. Sameshima S, Hasegawa E, Kitade O, Minaka N, Matsumoto $\mathrm{T}$ (1999) Phylogenetic comparison of endosymbionts with their host ants based on molecular evidence. Zool Sci 16:993-1000. doi:10.2108/zsj.16.993

16. Wernegreen JJ, Kauppinen SN, Brady SG, Ward PS (2009) One nutritional symbiosis begat another: phylogenetic evidence that the ant tribe Camponotini acquired Blochmannia by tending sap-feeding insects. BMC Evol Biol 9:292. doi:10.1186/1471-2148-9-292

17. Brown BP, Wernegreen JJ (2016) Deep divergence and rapid evolutionary rates in gut-associated Acetobacteraceae of ants. BMC Microbiol 16:140. doi:10.1186/s12866-016-0721-8

18. Hölldobler B, Wilson E (1990) The ants. Springer, Berlin

19. Ramalho MO, Martins C, Silva LMR, Martins VG, Bueno OC (2016) Molecular profile of the brazilian weaver ant Camponotus textor Forel (Hymenoptera, Formicidae). Neotrop Entomol 45:463-470. doi:10.1007/s13744-016-0392-z

20. Schremmer F (1979) Die Nahezu unbekannte neotropische weberameise Camponotus (Myrmobrachys) senex (Hymenoptera: Formicidae). Entomol Gen 5:363-378

21. Ramalho MO, Santos RM, Fernandes TT, Morini MSC, Bueno OC (2016) Cytochrome c oxidase I DNA sequence of Camponotus ants with different nesting strategies is a tool for distinguishing between morphologically similar species. Genetica 144:375383. doi:10.1007/s10709-016-9906-1

22. Longino JT (2006) New species and nomenclatural changes for the Costa Rican ant fauna (Hymenoptera: Formicidae). Myrmecol Nachr 8:131-143

23. Santos JC, Del-Claro K (2009) Ecology and behaviour of the weaver ant Camponotus (Myrmobrachys) senex. J Nat Hist 43:1423-1435. doi:10.1080/00222930902903236

24. Zara FJ, Bution ML Caetano FH (2010) Post-embryonic development of larvae of the weaver ant Camponotus textor (Hymenoptera: Formicidae). Sociobiology 55:557-578

25. Martins C, Souza RF, Bueno OC (2012) Presence and distribution of the endosymbiont Wolbachia among Solenopsis spp. (Hymenoptera: Formicidae) from Brazil and its evolutionary history. J Invertebr Pathol 109:287-296. doi:10.1016/j. jip.2012.01.001

26. Souza FR, Ramalho JDS, Morini MSC, Wolff JLC, Araújo RC, Mascara D (2009) Identification and characterization of Wolbachia in Solenopsis saevissima fire ants (Hymenoptera: Formicidae) in Southeastern Brazil. Curr Microbiol 58:189-194. doi:10.1007/s00284-008-9301-y

27. Frost CL, Fernández-Marín H, Smith JE, Hughes WOH (2010) Multiple gains and losses of Wolbachia symbionts across a tribe of fungus-growing ants. Mol Ecol 19:4077-4085. doi:10.1111/j.1365-294X.2010.04764.x

28. Braig HR, Zhou W, Dobson SL, O'Neill SL (1998) Cloning and characterization of a gene encoding the major surface protein of the bacterial endosymbiont Wolbachia pipientis. J Bacteriol 180:2373-2378

29. Shoemaker DD, Ross KG, Keller L, Vargo EL, Werren JH (2000) Wolbachia infections in native and introduced populations of fire ants (Solenopsis spp.). Insect Mol Biol 9:661-673. doi:10.1046/j.1365-2583.2000.00233.x

30. Baldo L, Hotopp JCD, Jolley KA, Bordenstein SR, Biber AA, Choudhury RR, Hayashi C, Maiden MCJ, Tettelin H, Werren JH (2006) Multilocus sequence typing system for the endosymbiont Wolbachia pipientis. Appl Environ Microbiol 72:7098-7110. doi:10.1128/AEM.00731-06

31. Zhou C, Yang Y, Jong A (1990) Mini-prep in ten minutes. Biotechniques 8:172-173

32. Bandelt HJ, Forster P, Röhl A (1999) Median-joining networks for inferring intraspecific phylogenies. Mol Biol Evol 16:37-48

33. Oksanen J, Kindt R, Legendre P, O'Hara B (2007) The vegan package. Commun Ecol 10:631-637

34. R Development Core Team (2016) R: a language and environment for statistical computing. http://www.R-project.org/

35. Bivand R, Pebesma E, Gomez-Rubio V (2013) Spatial data import and export. In: Applied spatial data analysis with R. Springer, New York

36. Kimura M (1980) A simple method for estimating evolutionary rates of base substitutions through comparative studies of nucleotide sequences. J Mol Evol 16:111-120. doi:10.1007/ BF01731581

37. Swofford DL (2002) PAUP* version 4.0. Phylogenetic analysis using parsimony (and other methods)

38. Hall T (1999) BioEdit: a user-friendly biological sequence alignment editor and analysis program for Windows 95/98/NT. Nucleic Acids Symp Ser 41:95-98

39. Higgins D, Bleasby A, Fuchs R (1992) CLUSTAL V: improved software for multiple sequence alignment. Comput Appl Biosci 8:189-191

40. Posada D, Crandall K (1998) Modeltest: testing the model of DNA substitution. Bioinformatics 14:817-818

41. Huelsenbeck J, Ronquist F (2001) MRBAYES: Bayesian inference of phylogenetic trees. Bioinformatics 17:754-755

42. Wenseleers T, Sundström L, Billen J (2002) Deleterious Wolbachia in the ant Formica truncorum. Proc R Soc Lond B 269:623-629. doi:10.1098/rspb.2001.1927

43. Degnan PH, Lazarus AB, Brock CD, Wernegreen JJ (2004) Host-symbiont stability and fast evolutionary rates in an antbacterium association: cospeciation of Camponotus species and their endosymbionts, Candidatus Blochmannia. Syst Biol 53:95-110

44. Buchner P (1965) Endosymbiosis of animals with plant microorganisms. Interscience Publishers, New York

45. Sauer C, Dudaczek D, Hölldobler B, Gross R (2002) Tissue localization of the endosymbiotic bacterium Candidatus Blochmannia floridanus in adults and larvae of the carpenter ant Camponotus floridanus. Appl Environ Microbiol 68:4187-4193. doi:10.1128/AEM.68.9.4187-4193.2002 
46. Schröder D, Deppisch H, Obermayer M, Krohne G, Stackebrandt E, Hölldobler B, Goebel W, Gross R (1996) Intracellular endosymbiotic bacteria of Camponotus species (carpenter ants): systematics, evolution and ultrastructural characterization. Mol Microbiol 21:479-489. doi:10.1111/j.1365-2958.1996.tb02557.x

47. Dykhuizen DE, Green L (1991) Recombination in Escherichia coli and the definition of biological species. J Bacteriol 173:7257-7268. doi:10.1128/JB.173.22.7257-7268.1991

48. Williams LE, Wernegreen JJ (2015) Genome evolution in an ancient bacteria-ant symbiosis: parallel gene loss among Blochmannia spanning the origin of the ant tribe Camponotini. PeerJ 3:e881. doi:10.7717/peerj.881

49. Dedeine F, Ahrens M, Calcaterra L, Shoemaker DD (2005) Social parasitism in fire ants (Solenopsis spp.): a potential mechanism for interspecies transfer of Wolbachia. Mol Ecol 14:1543-1548

50. Viljakainen L, Reuter M, Pamilo P (2008) Wolbachia transmission dynamics in Formica wood ants. BMC Evol Biol 8:55. doi:10.1186/1471-2148-8-55

51. Campbell MA, Van Leuven JT, Meister RC, Carey KM, Simon C, McCutcheon JP (2015) Genome expansion via lineage splitting and genome reduction in the cicada endosymbiont Hodgkinia. Proc Natl Acad Sci USA 112:10192-10199. doi:10.1073/ pnas. 1421386112

52. Watanabe M, Tagami Y, Miura K, Kageyama D, Stouthamer R (2012) Distribution patterns of Wolbachia endosymbionts in the closely related flower bugs of the genus Orius: implications for coevolution and horizontal transfer. Microb Ecol 64:537-545. doi:10.1007/s00248-012-0042-x

53. Jaenike J, Stahlhut JK, Boelio LM, Unckless RL (2010) Association between Wolbachia and Spiroplasma within Drosophila neotestacea: an emerging symbiotic mutualism? Mol Ecol 19:414-425. doi:10.1111/j.1365-294X.2009.04448.x

54. Baldo L, Werren JH (2007) revisiting Wolbachia supergroup typing based on wsp: spurious lineages and discordance with MLST. Curr Microbiol 55:81-87. doi:10.1007/s00284-007-0055-8

55. Jiggins FM (2003) Male-killing Wolbachia and mitochondrial DNA: selective sweeps, hybrid introgression and parasite population dynamics. Genetics 164:5-12

56. Raychoudhury R, Baldo L, Oliveira DCSG, Werren JH (2009) Modes of acquisition of Wolbachia: horizontal transfer, hybrid introgression, and codivergence in the Nasonia species complex. Evolution 63:165-183. doi:10.1111/j.1558-5646.2008.00533.x

57. Salunke BK, Salunkhe RC, Dhotre DP, Walujkar SA, Khandagale AB, Chaudhari R, Chandode RK, Ghate HV, Patole MS, Werren JH, Shouchea YS (2012) Determination of Wolbachia diversity in butterflies from Western Ghats, India, by a multigene approach. Appl Environ Microbiol 78:4458-4467. doi:10.1128/ AEM.07298-11 